\title{
Polymer flexibility and turbulent drag reduction
}

\author{
J. J. J. Gillissen \\ Kramers Laboratorium voor Fysische Technologie, J.M. Burgers Centre for Fluid Mechanics, \\ Delft University of Technology, Prins Bernhardlaan 6, 2628 BW Delft, The Netherlands \\ (Received 11 April 2008; revised manuscript received 30 July 2008; published 24 October 2008)
}

\begin{abstract}
Polymer-induced drag reduction is the phenomenon by which the friction factor of a turbulent flow is reduced by the addition of small amounts of high-molecular-weight linear polymers, which conformation in solution at rest can vary between randomly coiled and rodlike. It is well known that drag reduction is positively correlated to viscous stresses, which are generated by extended polymers. Rodlike polymers always assume this favorable conformation, while randomly coiling chains need to be unraveled by fluid strain rate in order to become effective. The coiling and stretching of flexible polymers in turbulent flow produce an additional elastic component in the polymer stress. The effect of the elastic stresses on drag reduction is unclear. To study this issue, we compare direct numerical simulations of turbulent drag reduction in channel flow using constitutive equations describing solutions of rigid and flexible polymers. When compared at constant $\phi r^{2}$, both simulations predict the same amount of drag reduction. Here $\phi$ is the polymer volume fraction and $r$ is the polymer aspect ratio, which for flexible polymers is based on average polymer extension at the channel wall. This demonstrates that polymer elasticity plays a marginal role in the mechanism for drag reduction.
\end{abstract}

DOI: 10.1103/PhysRevE.78.046311

PACS number(s): 47.50.-d, 47.57.Ng, 47.85.1b, 47.27.nd

\section{INTRODUCTION}

Turbulence puts severe limitations on fluid transport processes, and it is therefore of practical relevance that the addition of small concentrations $\left(\sim 10^{-5}\right.$ in weight $)$ of highmolecular-weight $\left(\sim 10^{6} \mathrm{~g} \mathrm{~mol}^{-1}\right)$ linear polymers to turbulent flow inhibits turbulent momentum transfer and consequently induces a reduction of the drag [1]. In addition to economic advantages, polymer-induced drag reduction is interesting from a fundamental point of view and many scientific efforts have been devoted to gaining a deeper understanding of wall-bounded turbulence and polymer dynamics [2-6].

Owing to their large molecular weight, polymers that are effective in drag reduction are usually highly flexible. In solution at rest, these flexible chains assume a randomly coiled configuration and in a statistical sense can be thought of as spherical particles. Exceptions are polyelectrolytes in deionized water [7] or polymers with a helical backbone structure [8]. Owing to a negligible level of flexibility, these polymers assume an extended conformation in solution at rest. Reference [9] induced drag reduction using polymers with varying flexibility. An increase in drag reduction with decreasing flexibility was observed, and it was concluded that the extended conformation is responsible for drag reduction. A similar conclusion was drawn in [10], in which a numerical simulation was performed of drag reduction by rigid polymers. Drag reduction was observed to diminish after the polymer model was modified to account for polymer flexibility.

In order for a flexible polymer to become an effective drag reducer, it needs to be unraveled to an extended conformation by the action of the fluid rate of strain. This phenomenon is reflected by the so-called onset phenomenon. Drag reduction sets in above an onset Reynolds number corresponding to a critical rate of strain required to unravel the flexible polymers [3]. Since rigid polymers are always in the favorable extended conformation, no onset phenomenon is observed. Instead they induce a more or less constant drag reduction efficiency as a function of the Reynolds number.

Drag reduction is the effect of forces between the polymers and the solvent molecules, referred to as polymer stresses. In general, polymers induce so-called viscoelastic stresses. In addition to having a capacity for dissipating mechanical energy, polymers have a capacity for storing energy, like springs. The elastic component of the polymer stress is related to polymer flexibility. When subjected to a varying fluid rate of strain, flexible polymers tend to stretch and coil, storing and releasing fluid mechanical energy. Rigid polymers, on the other hand, have a negligible level of flexibility and therefore induce purely viscous stresses. The viscous stress induced by polymers has an anisotropic character. This means that the magnitude of the viscosity induced by the polymers, referred to as the polymer viscosity, depends on the alignment of the polymer with respect to the fluid rate of strain. In simple shear flow, polymers tend to align in the flow direction. Since the polymers experience no fluid deformation along this direction, the polymer stress is negligible in the viscous sublayer of a turbulent boundary layer. Further away from the wall, the polymers experience straining motion, resulting in large polymer viscosity. Lumley [11] reasoned that the combination of an increased viscosity in the turbulent region and a negligible effect in the viscous layer results in a thickening of the viscous layer, with the net effect being a reduced drag coefficient. Benzi et al. [6] quantified these ideas and formulated a model for drag reduction, based on the Reynolds averaged equations of fluid momentum, turbulent kinetic energy, and polymer stress, where the latter was represented by a polymer viscosity.

As previously mentioned, a viscous representation for the polymer stress is not complete. In general, flexible polymers induce not only viscous stresses but also elastic stresses. A mechanism for drag reduction based on elastic stresses was put forward by De Gennes [4], who assumed that the polymers absorb energy above a certain cutoff frequency, corre- 
sponding to the polymer relaxation time. Unfortunately, these ideas have not been validated experimentally or numerically and therefore remain speculative.

In this work, we aim to clarify the role of elastic polymer stresses on drag reduction. We use direct numerical simulations (DNS) to study drag reduction induced by polymers having different levels of flexibility. For this purpose, we use constitutive equations representing solutions of rigid and elastic dumbbells [12]. Owing to the hypothetical nature of these representations, the simulations cannot be used for quantitative predictions. However, the equations are considered conceptually correct and the predicted flow properties are in qualitative agreement with experiments. The simplicity of the equations allows us to perform simulations using relatively little computer resources. Therefore, a large number of simulations could be conducted, such that the effect of polymer flexibility could be explored over a wide parameter range.

\section{NUMERICAL MODEL}

\section{A. Fluid}

We consider polymer-induced drag reduction in turbulent channel flow, where a fluid of density $\rho$ is driven by a constant pressure gradient $-d \bar{\Pi} / d x$ between two no-slip walls separated in the $y$ direction by a distance $D$. Periodic boundary conditions are imposed in the streamwise $(x)$ and spanwise $(z)$ directions. The flow is governed by the incompressible Navier-Stokes equations, supplemented by the divergence of the polymer stress tensor $\tau$,

$$
\begin{gathered}
\rho \frac{D \boldsymbol{u}}{D t}=\nabla \cdot(-\Pi \delta+2 \mu S+\tau), \\
\boldsymbol{\nabla} \cdot \boldsymbol{u}=0 .
\end{gathered}
$$

Here $\mathbf{u}$ is the fluid velocity vector, $t$ is time, $\boldsymbol{\nabla}$ is the nabla operator, $\boldsymbol{\delta}$ is the unit tensor, $D / D t=\partial / \partial t+\mathbf{u} \cdot \boldsymbol{\nabla}$ is the material derivative, $\mathbf{S}=\frac{1}{2}\left(\nabla \mathbf{u}^{T}+\nabla \mathbf{u}\right)$ is the rate of strain tensor, $\Pi$ is the pressure, $\mu=\nu \rho$ is the solvent dynamic viscosity, and $\nu$ is the solvent kinematic viscosity. Without polymers $\boldsymbol{\tau}=\mathbf{0}$, the flow is fully characterized by the Reynolds number $\operatorname{Re}_{\tau}$ $=U_{\tau} D / \nu$ based on the friction velocity $U_{\tau}$ $=\left[-\frac{1}{2}(d \bar{\Pi} / d x)(D / \rho)\right]^{1 / 2}$.

We use a pseudospectral flow solver. Spatial derivatives are computed with a Fourier basis for the wall-parallel directions and a second-order, central, finite-differences scheme for the wall-normal direction. Time integration is achieved with the second-order, explicit Adams-Bashforth scheme, except for the nonlinear term on the right-hand side of Eq. (3b) (given below), which is treated with the second-order, implicit Crank-Nicholson scheme [13].

We conducted one simulation of a Newtonian flow, and several simulations of drag-reduced flow. For the Newtonian flow, the channel dimensions and number of grid points are $3 D \times D \times 1.5 D$ and $96 \times 128 \times 96$ in the $x, y$, and $z$ direction. To capture the larger near-wall vortical structures in the dragreduced flows, we use larger dimensions $4.5 D \times D \times 2.25 D$ and coarser resolutions $96 \times 96 \times 96$. To study the role of polymer flexibility on drag reduction, we conducted simulations using two different constitutive equations describing solutions of rigid and flexible polymers.

\section{B. Rigid polymers}

Rigid polymers are modeled as massless, neutrally buoyant, non-Brownian, noninteracting rigid dumbbells, which are smaller than the Kolmogorov length scale [12]. The rigid dumbbell displayed in Fig. 1(a) consists of two spheres of diameter $d$ that are connected by a rigid rod of length $l$. The spheres interact with the flow by Stokes forces. The dumbbell volume fraction is $\phi$ and the aspect ratio is $r=l / d$. The stress generated by the dumbbells $\tau$ is a viscous stress, with viscosity depending on the orientation of the dumbbells relative to the axes of fluid deformation,

$$
\tau=\frac{9}{2} \mu \phi r^{2} S:\langle p p p p\rangle .
$$

Here $\boldsymbol{p}$ is the polymer orientation unit vector and $\langle\cdots\rangle$ is an average over polymers contained in a (small) volume centered at the point where the stress is to be determined. The concentration parameter $\phi r^{2}$ measures the importance of the polymer stress relative to the Newtonian stress and determines the amount of drag reduction.

Computing the stress requires the fourth-order moment of the polymer orientation distribution function $\langle\boldsymbol{p p p p}\rangle$. We approximate this quantity by solving the equation of change for the second-order moment $\langle p \boldsymbol{p}\rangle$,

$$
\frac{D\langle p p\rangle}{D t}-\nabla \boldsymbol{u}^{T} \cdot\langle\boldsymbol{p} \boldsymbol{p}\rangle-\langle\boldsymbol{p} \boldsymbol{p}\rangle \cdot \nabla \boldsymbol{u}+2 \boldsymbol{\nabla} \boldsymbol{u}:\langle\boldsymbol{p} p \boldsymbol{p} \boldsymbol{p}\rangle=\kappa \nabla^{2}\langle\boldsymbol{p} \boldsymbol{p}\rangle,
$$

and applying the closure developed in [14] to express $\langle\boldsymbol{p p p p}\rangle$ in terms of $\langle\boldsymbol{p} \boldsymbol{p}\rangle$. This closure model relates the principal values of $\langle\boldsymbol{p} p \boldsymbol{p} \boldsymbol{p}\rangle$ to the principal values of $\langle\boldsymbol{p} \boldsymbol{p}\rangle$ by means of a fit to exact solutions. The fit coefficients are constrained to reproduce correct $\langle\boldsymbol{p p p p}\rangle$ for the three limiting cases of isotropic, biaxial, and uniaxial distribution functions.

The diffusive term $\kappa \nabla^{2}\langle p \boldsymbol{p}\rangle$ models unresolved spatial variations where $\kappa$ is the artificial diffusivity [15].

\section{Flexible polymers}

In addition to rigid polymers, we have simulated drag reduction by flexible polymers. Flexible polymers are modeled as FENE-P (finitely extensible nonlinear elastic with the Peterlin approximation) dumbbells [12]. As drawn in Fig. 1(b), the elastic dumbbell consists of two spheres of diameter $d$, which are separated by a vector $\boldsymbol{l}$ and connected by a FENE spring, which accounts for the tendency of Brownian fluctuations to drive the polymer to its equilibrium coiled configuration. The maximum and equilibrium lengths of the spring are $l_{\max }$ and $l_{\text {eq. }}$. As for rigid dumbbells, the flexible dumbbells are assumed massless, noninteracting, and smaller than the Kolmogorov length scale. The polymer stress, 


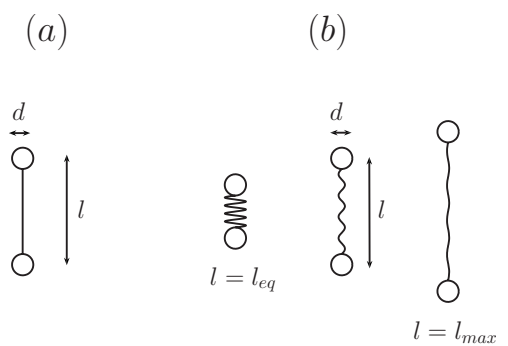

FIG. 1. Rigid dumbbell (a) and elastic dumbbell at three levels of extension (b)

$$
\boldsymbol{\tau}=\frac{9 \mu \phi r_{\mathrm{eq}}^{2}}{4 \lambda}\left(\frac{c}{1-\frac{\operatorname{tr} c}{b}}-\delta\right)
$$

is determined by the conformation tensor $\boldsymbol{c}=\langle\boldsymbol{l l}\rangle / l_{\mathrm{eq}}^{2}$, which evolves according to

$$
\frac{D c}{D t}-\nabla \boldsymbol{u}^{T} \cdot \boldsymbol{c}-\boldsymbol{c} \cdot \boldsymbol{\nabla} \boldsymbol{u}=\frac{1}{\lambda}\left(\boldsymbol{\delta}-\frac{c}{1-\frac{\operatorname{tr} c}{b}}\right)+\kappa \nabla^{2} \boldsymbol{c} .
$$

The polymer stress is determined by three dimensionless parameters: the concentration parameter at equilibrium $\phi r_{\mathrm{eq}}^{2}$ $=\phi l_{\mathrm{eq}}^{2} / d^{2}$, the extensibility parameter $b=l_{\mathrm{max}}^{2} / l_{\mathrm{eq}}^{2}$, and the Weissenberg number $\mathrm{Wi}_{\tau}=\gamma \lambda$, where $\gamma=U_{\tau}^{2} / \nu$ is the mean shear rate at the wall and $\lambda=\frac{3 \pi}{4} \mu d l_{\mathrm{eq}}^{2} / k_{B} T$ is the polymer relaxation time, with $k_{B}$ the Boltzmann constant and $T$ the temperature. For small $\mathrm{Wi}_{\tau}$, the polymers are randomly coiled. The conformation tensor is isotropic: $\boldsymbol{c}=\boldsymbol{\delta}$, and the
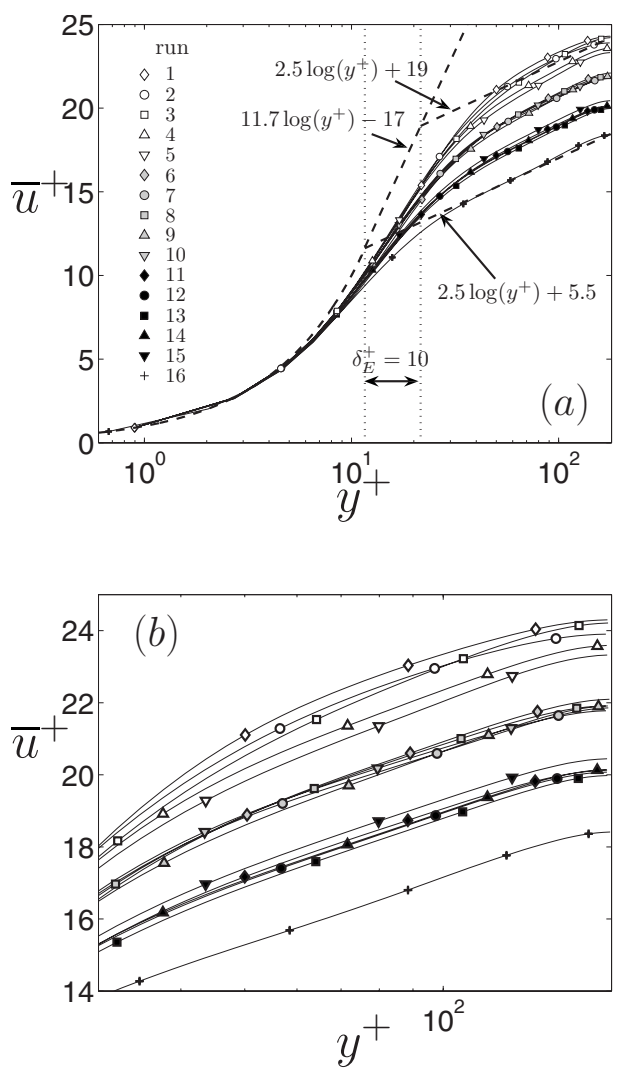

FIG. 2. Mean flow as a function of the distance to the wall. Comparison between Newtonian flow, drag-reduced flow with rigid polymers and drag-reduced flow with flexible polymers. As explained in the legend, the different symbols correspond to the different simulations. The parameters are listed in Table I.

TABLE I. Parameters used in the simulations. Types $N, R$, and $E$ correspond to simulations of Newtonian flow, drag-reduced flow using rigid dumbbells, and drag-reduced flow using elastic dumbbells. For type $E$, the value given for $\frac{9}{4} \phi r_{\text {eff }}^{2}$ is based on the Reynolds average at $y^{+}=0$, as given by Eq. (7).

\begin{tabular}{lccccccc}
\hline \hline Run & Type & $\frac{9}{4} \phi r_{\mathrm{eff}}^{2}$ & $\mathrm{Wi}_{\tau}$ & $\frac{9}{4} \phi r_{\mathrm{eq}}^{2}$ & $\kappa^{+}$ & $R_{D}$ & $\delta_{E}^{+}$ \\
\hline 1 & $R$ & 50.0 & & & 1.5 & 0.44 & 10.0 \\
2 & $E$ & 49.3 & 1500 & 0.0052 & 3.0 & 0.42 & 9.3 \\
3 & $E$ & 46.5 & 450 & 0.0053 & 3.0 & 0.42 & 9.3 \\
4 & $E$ & 44.2 & 150 & 0.0061 & 1.5 & 0.39 & 8.1 \\
5 & $E$ & 37.2 & 50 & 0.011 & 1.5 & 0.38 & 7.5 \\
6 & $E$ & 25.0 & & & 1.5 & 0.31 & 5.3 \\
7 & $E$ & 24.9 & 1500 & 0.0026 & 3.0 & 0.30 & 5.1 \\
8 & $E$ & 23.8 & 450 & 0.0027 & 3.0 & 0.31 & 5.3 \\
9 & $E$ & 22.2 & 150 & 0.0031 & 1.5 & 0.30 & 5.0 \\
10 & $E$ & 22.0 & 50 & 0.0054 & 1.5 & 0.30 & 5.2 \\
11 & $R$ & 10.0 & & & 1.5 & 0.18 & 2.4 \\
12 & $E$ & 10.1 & 1500 & 0.0010 & 6.0 & 0.17 & 2.3 \\
13 & $E$ & 9.9 & 450 & 0.0011 & 6.0 & 0.16 & 2.1 \\
14 & $E$ & 9.8 & 150 & 0.0012 & 3.0 & 0.17 & 2.3 \\
15 & $E$ & 11.2 & 50 & 0.0022 & 2.0 & 0.20 & 2.8 \\
16 & $N$ & & & & & & \\
\hline \hline
\end{tabular}



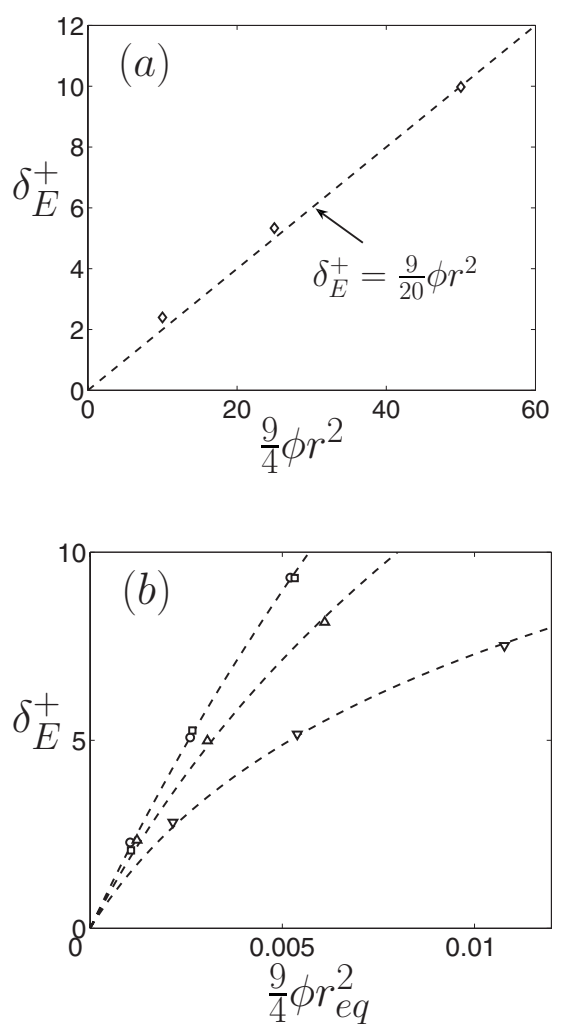

FIG. 3. (a) Elastic layer thickness $\delta_{E}^{+}$versus concentration parameter $\phi r^{2}$ for rigid dumbbells. (b) Elastic layer thickness $\delta_{E}^{+}$versus equilibrium concentration parameter $\phi r_{\mathrm{eq}}^{2}$ for elastic dumbbells having $\mathrm{Wi}_{\tau}=50$ (downward triangles), $\mathrm{Wi}_{\tau}=150$ (upward triangles), $\mathrm{Wi}_{\tau}=450$ (squares), and $\mathrm{Wi}_{\tau}=1500$ (circles).

polymer stress attains the form of a Newtonian viscous stress: $\boldsymbol{\tau}=\frac{9}{2} \mu \phi r_{\mathrm{eq}}^{2} \boldsymbol{S}$. The resulting flow is equivalent to a Newtonian flow, with an increased viscosity. Obviously there will be no drag reduction, but rather a drag increase. Significant drag reduction requires the polymers to be extended, which happens when $\mathrm{Wi}_{\tau} \gg 1$.

\section{Parameters}

We have conducted one simulation of Newtonian (polymer-free) flow, three simulations of drag-reduced flow using rigid polymers, and twelve simulations of dragreduced flow using flexible polymers. The Reynolds number was always $\operatorname{Re}_{\tau}=360$.

In the rigid polymer solutions, the concentration parameter was chosen to be $\frac{9}{4} \phi r^{2}=10,25$, and 50 , resulting in approximately $0.2,0.3$, and $0.4 \mathrm{drag}$ reduction $R_{D}$, defined as the relative decrease of the friction factor $f$,

$$
R_{D}=1-\frac{f^{P}}{f^{N}} .
$$

Here $f^{P}$ and $f^{N}$ correspond to the polymer solution flow and the Newtonian flow, respectively. The friction factor is related to the nondimensional bulk velocity $U^{+}$as $1 / \sqrt{f}=U^{+}$, which is obtained by averaging the simulated velocity profiles over the nondimensional channel height $D^{+}=\operatorname{Re}_{\tau}$ $=D U_{\tau} / \nu$,

$$
U^{+}=\frac{2}{\operatorname{Re}_{\tau}} \int_{0}^{\operatorname{Re}_{\tau} / 2} \bar{u}^{+} d y^{+} .
$$

It is noted that variables indicated with the superscript + are given in wall units, being scaled with the friction velocity $U_{\tau}$ and the solvent kinematic viscosity $\nu$.

In the flexible polymer solutions, the extensibility parameter was always $b=10^{4}$, corresponding to a polymerization index of $N \sim 10^{5}$, typical for polymers in drag reduction experiments [3]. Different levels of polymer flexibility were studied by varying the Weissenberg number $\mathrm{Wi}_{\tau}$ over a 30 fold range: $50,150,450$, and 1500 . For each $\mathrm{Wi}_{\tau}$, three different values for the equilibrium concentration parameter $\phi r_{\text {eq }}^{2}$ were studied, inducing $R_{D} \approx 0.2,0.3$, and 0.4 , similar to that for the rigid dumbbell simulations. Table I summarizes the values used for the model parameters and $R_{D}$. Values for $\kappa$, used in Eqs. (2b) and (3b), were chosen as small as possible while maintaining a smooth and stable numerical solution.

\section{RESULTS}

The mean velocity profiles $\bar{u}^{+}\left(y^{+}\right)$for all simulations are shown in Fig. 2(a). For clarity, we show the inertial layer in detail in Fig. 2(b). As explained in the legend of Fig. 2(a), the symbols correspond to the different simulations. The symbols are superimposed on the lines for only one-tenth of all grid points. The Newtonian profile (run 16) is linear for $y^{+} \lesssim 10$ and logarithmic with slope 2.5 for $y^{+} \gtrsim 10$, referred to as the viscous layer and the inertial layer, respectively. In the polymer solution flows, the observed increase in nondimensional velocity is equivalent to a reduced friction factor $f$. The increased velocity is realized by an upward, parallel shift of the inertial layer, due to the formation of a third layer in between the viscous layer and the inertial layer. With increasing $R_{D}$, the velocity profile in this intermediate layer approaches a logarithm of slope 11.7, asymptotically.

This intermediate, logarithmic layer was discovered experimentally and was termed the elastic layer [3]. According to this concept, the velocity profile in drag-reduced flow can be described by a three-layer model,

$$
\bar{u}^{+}= \begin{cases}y^{+} & \text {if } 0<y^{+}<11.6 \\ 11.7 \log y^{+}-17 & \text { if } 11.6<y^{+}<11.6+\delta_{E}^{+} \\ 2.5 \log y^{+}+9.2 \log \left(11.6+\delta_{E}^{+}\right)-17 & \text { if } 11.6+\delta_{E}^{+}<y^{+}<\operatorname{Re}_{\tau} / 2\end{cases}
$$




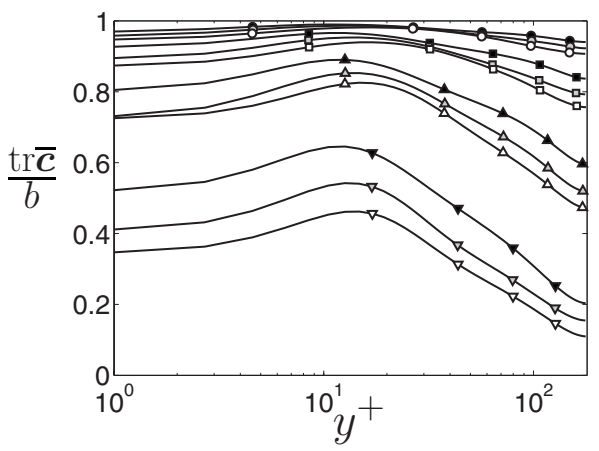

FIG. 4. Polymer extension normalized by maximum extension as a function of the distance to the wall. Symbols correspond to different simulations as explained in Fig. 2(a).

From the wall outward, these layers are referred to as the viscous layer, the elastic layer, and the inertial layer. In the following, we use the elastic layer thickness $\delta_{E}^{+}$to quantify the amount of drag reduction and explore its dependence on the various polymeric properties. We compute $\delta_{E}^{+}$(numerically) from the equation between the simulated bulk velocity and the parametrized bulk velocity, where the latter is defined as the average of Eq. (9) over the channel cross section.

In Fig. 3(a), $\delta_{E}^{+}$is plotted versus $\phi r^{2}$ for the rigid polymer cases (runs 1, 6, and 11). The linear relation indicates that the drag reduction efficiency per polymer, measured by $\delta_{E}^{+} / \phi r^{2}$, is independent of the amount of drag reduction.

For flexible polymers, the situation is more complicated. Besides polymer concentration $\phi r_{\mathrm{eq}}^{2}$, there is an additional dependency on polymer flexibility $\mathrm{Wi}_{\tau}$. Figure $3(\mathrm{~b})$ presents the data in the $\left(\phi r_{\mathrm{eq}}^{2}, \delta_{E}^{+}\right)$plane. The dashed curves connect points of constant $\mathrm{Wi}_{\tau}$. The data for $\mathrm{Wi}_{\tau}=450$ and 1500 collapse on a single curve, which is linear in good approximation. This implies that for $\mathrm{Wi}_{\tau}>450$, elastic dumbbells behave as rigid dumbbells. This observation is understood by considering the level of polymer extension, which is shown in Fig. 4. It is seen that for $\mathrm{Wi}_{\tau}=450$ and 1500 , the polymers are nearly fully stretched and therefore behave as rigid rods.

For smaller $\mathrm{Wi}_{\tau}$, the data in the $\left(\phi r_{\mathrm{eq}}^{2}, \delta_{E}^{+}\right)$plane deviate from the rodlike, linear relation. As the concentration increases, the flow becomes more drag-reduced, and less effective in stretching polymers, as can be seen in Fig. 4. Apparently, a decrease in polymer extension is related to a decrease in the drag reduction efficiency per polymer, measured by $\delta_{E}^{+} /\left(\phi r_{\mathrm{eq}}^{2}\right)$.

To quantify this relation, we define an effective concentration parameter based on polymer extension at the wall,

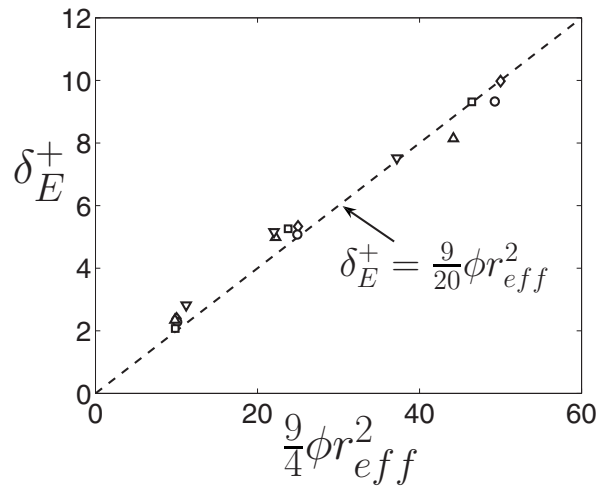

FIG. 5. Elastic layer thickness $\delta_{E}^{+}$versus effective concentration parameter $\phi r_{\text {eff }}^{2}$, for rigid dumbbells (diamonds) and elastic dumbbells having $\mathrm{Wi}_{\tau}=50$ (downward triangles), $\mathrm{Wi}_{\tau}=150$ (upward triangles), $\mathrm{Wi}_{\tau}=450$ (squares), and $\mathrm{Wi}_{\tau}=1500$ (circles).

$$
\phi r_{\mathrm{eff}}^{2}=\left.\phi \overline{r^{2}}\right|_{y^{+}=0}=\left.\phi r_{\mathrm{eq}}^{2} \operatorname{tr} \overline{\boldsymbol{c}}\right|_{y^{+}=0} .
$$

In Fig. 5, we plot our results in the $\left(\phi r_{\mathrm{eff}}^{2}, \delta_{E}^{+}\right)$plane for both elastic and rigid dumbbells. It is noted that for rigid dumbbells, the aspect ratio is fixed: $\phi r_{\text {eff }}^{2}=\phi r^{2}$. A single linear relationship is seen to reasonably fit all the data. Apparently elastic dumbbells and rigid dumbbells induce identical drag reduction when compared at equal $\phi r_{\text {eff. }}^{2}$. It is therefore concluded that the amount of drag reduction is related to the average polymer extension near the wall, while fluctuations have little or no effect. This indicates that elastic stresses have a marginal influence in the drag reduction mechanism.

\section{CONCLUSIONS}

We have used DNS to study drag reduction in turbulent channel flow induced by rigid and elastic polymers. In both systems, drag reduction depends similarly on $\phi r^{2}$. Here $\phi$ is the polymer volume concentration and $r$ is the polymer aspect ratio, which for flexible polymers is based on average polymer extension at the wall.

This similarity indicates that the mechanism for drag reduction is nearly identical for flexible and rigid polymers, and it is therefore due to the viscous polymer stresses. The elastic polymer stresses, owing to polymer coiling and stretching, seem to play a minor part.

Obtaining a predictive relation between drag reduction and polymeric properties requires an expression for $\phi r^{2}$ at the wall. An estimate could be obtained by assuming simple shear conditions. Unfortunately, the FENE-P model does not reproduce correct stress characteristics in simple shear flow, and therefore cannot be used for this purpose. Quantitative predictions as such must come from experiments.
[1] B. A. Toms, Proceedings of the International Congress on Rheology (North-Holland, Amsterdam, 1948), Vol. 2, p. 135.

[2] J. L. Lumley, Annu. Rev. Fluid Mech. 1, 367 (1969).

[3] P. Virk, J. Fluid Mech. 45, 417 (1971).
[4] P. G. De Gennes, Physica A 140, 9 (1986).

[5] G. Ryskin, Phys. Rev. Lett. 59, 2059 (1987).

[6] R. Benzi, E. De Angelis, V. S. L’vov, and I. Procaccia, Phys. Rev. Lett. 95, 194502 (2005). 
[7] S. Sasaki, J. Phys. Soc. Jpn. 60, 2613 (1991).

[8] S. Sasaki, J. Phys. Soc. Jpn. 60, 868 (1991).

[9] P. Virk, D. C. Sherman, and D. L. Wagger, AIChE J. 43, 3257 (1997).

[10] J. M. J. Den Toonder, M. A. Hulsen, G. D. C. Kuiken, and F. T. M. Nieuwstadt, J. Fluid Mech. 337, 193 (1997).

[11] J. L. Lumley, J. Polym. Sci. D: Macromol. Rev. 7, 263 (1973).

[12] R. B. Bird, O. Hassager, R. C. Armstrong, and C. F. Curtiss, Dynamics of Polymeric Liquids (Wiley, New York, 1977),
Vol. 2 .

[13] P. K. Ptasinski, B. J. Boersma, F. T. M. Nieuwstadt, M. A. Hulsen, B. H. A. van den Brule, and J. C. R. Hunt, J. Fluid Mech. 490, 251 (2003).

[14] E. D. Wetzel, Ph.D. thesis, University of Illinois at UrbanaChampaign (1999).

[15] J. J. J. Gillissen, B. J. Boersma, P. H. Mortensen, and H. I. Andersson, Phys. Fluids 19, 115107 (2007). 